\title{
REVISIONES
}

\section{El estudio de la segregación socioeconómica y académica de la educación chilena}

\author{
Study of socioeconomic and academic segregation in Chilean education \\ Estudo da segregação socioeconômica e acadêmica da educação chilena
}

\author{
Cristián Bellei $C$. \\ Centro de Investigación Avanzada en Educación, Departamento de Sociología, Universidad de Chile. Correo \\ electrónico: cbellei@ciae.uchile.cl. Telf.: (562) 9782779 - (562) 9782762
}

\begin{abstract}
RESUMEN
Este trabajo analiza la segregación de la población escolar en Chile en dos dimensiones: la segregación socioeconómica y la segregación académica, materia de enorme interés dado la fuerte configuración de mercado del sistema educacional chileno. El texto sitúa el análisis de la segregación en el marco de la creciente preocupación por la equidad educacional y propone una conceptualización que vincula la segregación escolar con la investigación acerca del "efecto de los compañeros" en educación (peer effects). A continuación, se revisa y discute la evidencia empírica disponible acerca de la magnitud, causas y efectos de la segregación socioeconómica y la segregación académica de la educación chilena. El trabajo concluye identificando algunos de los desafíos que este campo de estudio presenta a la investigación científica y proponiendo líneas de investigación futura que permitan avanzar en el conocimiento de un tema relevante pero comparativamente poco estudiado.
\end{abstract}

Palabras clave: segregación escolar, efecto de los compañeros, equidad educacional, sociología de la educación.

\begin{abstract}
This paper analyzes school segregation in Chile in two dimensions: socioeconomic segregation and academic segregation; a matter of great interest given the radical market oriented organization of the Chilean education. The analysis is situated in the context of the growing concern about the educational equity and proposes a conceptualization that links school segregation to the study of the peer-effect in education (i.e. the effect caused by school peers). Additionally, the paper reviews and discusses the available empirical evidence about the magnitude, causes and effects of the socioeconomic and academic segregation in Chile. Finally, the paper identifies some of the challenges that school segregation poses to the scientific research and proposes some research branches for additional studies, in order to advance in the academic knowledge of this relevant but poorly studied issue.
\end{abstract}

Key words: school segregation, peer effect, equity in education, sociology of education.

\section{RESUMO}

Analisa-se a segregação da população escolar no Chile em duas dimensões: a segregação socioeconômica e a acadêmica, tema de grande interesse, dada a forte configuração do mercado do sistema educacional chileno. Situa-se a análise da segregação no contexto da crescente preocupação com a equidade educacional e propõe-se uma conceituação que liga a segregação escolar à pesquisa sobre o "efeito de pares" na educação (peer effects). Revisa-se e situa-se a evidência empírica sobre a magnitude, causas e efeitos da segregação socioeconômica e da segregação acadêmica na educação chilena. Conclui-se identificando alguns dos desafios que este campo de estudo apresenta à pesquisa científica, propondo linhas para futura pesquisa que permita avançar no conhecimento de um tema relevante, porém pouco estudado.

Palavras chave: segregação escolar, efeito dos pares, equidade educacional, sociologia da educação. 


\section{LOS COMPAÑEROS DE ESCUELA Y LA EQUIDAD}

En las sociedades modernas los sistemas educacionales han sido definidos como una agencia clave en el proceso dual de socialización y selección técnica de las personas, especialmente en referencia al mercado laboral. La estratificación social basada en los sistemas de clases sociales considera al logro educacional simultáneamente como un predictor y como un componente de la posición de clase de un individuo. Este carácter medular de la educación para la estratificación social le ha situado al centro de las pretensiones de legitimación de las sociedades democráticas que aspiran a ser sociedades abiertas a la movilidad social: los gobernantes ofrecen igualdad de oportunidades en y a través de la educación casi en la misma medida en que los ciudadanos demandan un mayor acceso a ella.

El principio de equidad aplicado al campo de la educación institucional (junto con los requerimientos organizacionales de los sistemas masivos de educación escolar) ha derivado en un constante proceso de "estandarización" del servicio educacional: la organización basada en clases, la enseñanza distribuida en grados secuenciales, la escolaridad obligatoria, la profesionalización de los docentes, el currículum nacional obligatorio, el calendario escolar, los sistemas de evaluación estandarizados, etc. Para que la desigualdad de las estratificadas sociedades contemporáneas sea reconocida como legítima parece condición que el conjunto de los ciudadanos haya tenido una cierta "experiencia común" en su proceso formativo.

Ciertamente, no todos los aspectos de la educación son "estandarizables" y, por tanto, las experiencias escolares de los alumnos difieren en cierto grado inevitablemente. Una cuestión crítica a este respecto es el hecho de que los alumnos asisten prolongadamente (y en muchos casos, exclusivamente) a un mismo establecimiento escolar y son en esa medida afectados por las particularidades de la escuela o liceo al que asisten. Así, aunque dichos establecimientos deben regirse por ciertas normas comunes, se acepta también (y en muchos casos se valora) que posean características distintivas: ¿hasta qué punto dichas características no son más que rasgos anecdóticos o secundarios que no ponen en riesgo el principio de equidad educacional? Este trabajo analiza una de aquellas características reacias a la estandarización y que, sin embargo, afecta de un modo potencialmente relevante la experiencia formativa de los estudiantes: los alumnos que componen la escuela a la que se asiste, es decir, los compañeros.

Aunque intuitivamente todos reconocemos que los compañeros de escuela son un factor importante en moldear la experiencia formativa que se tiene, no es evidente que esta diferenciación esté relacionada con el principio de equidad educacional, en el sentido de afectar el grado en que cada alumno se beneficia (especialmente en términos instruccionales) de la educación. Esto por al menos dos razones concatenadas. En primer lugar, habría que demostrar que la productividad de la educación es diferente según los compañeros que co-habitan el aula-escuela. A continuación, habría que identificar patrones sistemáticos de distribución desigual de los alumnos entre escuelas que se vinculen de modo relevante con las diferencias de productividad antes referidas. Dicho coloquialmente: si lo que importa es el plan de estudios, el profesor y el trabajo que realiza cada alumno, ¿por qué preocuparse de quién más está en la lista del curso? Pero, aun en el caso de que los compañeros sean en cierto modo relevantes, ¿cómo podrían las familias evaluar 
anticipadamente el (eventual) efecto que los compañeros ejercerían sobre sus hijos y luego decidir dónde matricularlos para su mayor conveniencia?

La literatura académica internacional ha abordado estas dos cuestiones explorando el efecto que pudieran tener en los aprendizajes de los alumnos diferentes características de sus compañeros, principalmente el género, la raza, el nivel socioeconómico y la habilidad académica (en este trabajo nos ocuparemos solo de estas dos últimas). Esta línea de investigación ha sido articulada en torno a la pregunta por la existencia y eventuales características del "efecto de los compañeros" (peer effect). Complementariamente, existe una abundante literatura que aborda la cuestión de la distribución desbalanceada de los alumnos entre las escuelas, especialmente en términos de su raza y origen social. Esta línea de investigación se articula en torno a la noción de "segregación escolar". El objetivo de este trabajo es contribuir al estudio de la segregación socioeconómica y académica de la educación chilena, situando dicho análisis en el contexto de las dos líneas de investigación recién mencionadas.

En términos más específicos, el artículo comienza situando el análisis de la segregación en el marco de la preocupación por la equidad educacional y propone un marco analítico que vincula la segregación escolar con la investigación acerca del "efecto de los compañeros" en educación; a continuación, se revisa y discute la evidencia empírica disponible acerca de la magnitud, causas y efectos de la segregación socioeconómica y la segregación académica de la educación chilena. Finalmente, luego de resumir las principales conclusiones, el texto concluye discutiendo algunos de los desafíos que este campo presenta a la investigación científica y proponiendo líneas temáticas para futuros estudios que contribuyan a llenar los actuales vacíos de conocimiento. En efecto, la relevancia de este trabajo radica en que, aunque la segregación escolar despierta preocupación en muchos educadores y académicos vinculados al campo de la educación, esta ha sido relativamente postergada tanto por la investigación científica como por las prioridades de política educacional en Chile.

\section{EL "EFECTO DE LOS COMPAÑEROS" EN EDUCACIÓN}

La noción general detrás del efecto de los compañeros es que la composición de los alumnos que comparten un aula-escuela afecta los resultados educacionales obtenidos por dichos alumnos y, en consecuencia, que diferentes agrupaciones de estudiantes producirán logros educativos distintos. Dado que la literatura sobre el efecto de los compañeros se asocia con la investigación sobre factores explicativos de los logros escolares de los alumnos (siendo la composición del aula-escuela una de las variables explicativas de dicho resultado), desde el punto de vista de un alumno en particular se trata de estimar si existe un "efecto de los compañeros" sobre sus logros académicos.

La investigación empírica destinada a identificar y estimar el efecto de los compañeros sobre los resultados de aprendizaje de los alumnos enfrenta desafíos metodológicos de enorme complejidad, por lo que su progreso ha sido lento y dificultoso; tampoco la reflexión teórica ha hecho grandes avances en esta materia, lo que a su vez ha redundado en dificultades adicionales para interpretar y orientar la investigación empírica (ver por ejemplo la discusión de Thrupp, Lauder y Robinson, 2002). En principio, los compañeros de aula-escuela ejercen múltiples tipos de efectos sobre la educación de un alumno en 
particular, los cuales se pueden clasificar en efectos sobre el ambiente para el aprendizaje (contexto) y efectos sobre los procesos de enseñanza-aprendizaje; a su vez, es posible argumentar que dichos efectos pueden operar por canales diferentes, lo que permite clasificarlos en efectos directos sobre el alumno en cuestión y efectos indirectos o mediados por otros. Nótese que tanto los efectos sobre el ambiente como sobre los procesos pueden tener el carácter directo e indirecto, tal como se ejemplifica en el Cuadro 1.

Cuadro 1. Efecto de los compañeros: tipología de efectos

\begin{tabular}{|c|c|c|}
\hline & Sobre el ambiente & Sobre los procesos \\
\hline Efectos directos & Clima del aula & Grupo de estudio \\
& Disciplina escolar & Tutorías entre pares \\
\hline Efectos indirectos & $\begin{array}{c}\text { Disponibilidad de recursos } \\
\text { para el aprendizaje }\end{array}$ & $\begin{array}{c}\text { Expectativas del docente } \\
\text { Nivel de dificultad de la } \\
\text { clase }\end{array}$ \\
& $\begin{array}{c}\text { Apoyo de la comunidad } \\
\text { escolar a la labor educativa } \\
\text { de la escuela }\end{array}$ & \\
\hline
\end{tabular}

Fuente: elaboración propia; ver texto para definiciones.

Es importante tener claro que los efectos de "los compañeros" pueden ser de naturaleza facilitadora $\mathrm{u}$ obstaculizadora de la labor educativa y ejercer, por tanto, un efecto positivo o negativo sobre los logros escolares (algunos compañeros indisciplinados en el aula pueden afectar negativamente las oportunidades de aprendizaje del conjunto de la clase; un grupo de alumnos motivados puede disponer positivamente al profesor para con el curso en su totalidad). Finalmente, aunque es posible argumentar que parte del efecto de los compañeros se produce "mecánicamente" en los contextos naturales del aulaescuela (un grupo de alumnos gritando en un aula difícilmente pasará inadvertido para el resto), la investigación empírica (especialmente de naturaleza cualitativa en este caso) ha mostrado que una parte fundamental del efecto de los compañeros se "produce" (es decir, actualiza su potencial) a través de las interpretaciones y conductas de los profesores. ${ }^{1}$

La relación entre la composición de las aulas-escuelas (especialmente en términos sociales y raciales) y los procesos y resultados educacionales ha sido estudiada por la sociología de la educación al menos desde los años 1950 en Estados Unidos y desde los 1960 en Europa, especialmente en Francia e Inglaterra. Sin embargo, el estatus causal del efecto de los compañeros sobre los logros escolares ha sido mucho más difícil de probar

1 Wilkinson et al. (2002) ofrecen una discusión teórica y metodológica sobre el efecto de los compañeros que deriva en un modelo de análisis muy cercano al aquí presentado. Sin embargo, en ese mismo volumen -completamente dedicado a la discusión acerca del "efecto de los compañeros"- se realiza una distinción discutible entre "efectos de composición" y "efecto de los compañeros", definiendo-grosso modo-lo que hemos denominado "efectos indirectos" como "efectos de composición" y los "efectos directos" como "verdadero" efecto de los compañeros. A mi juicio, Wilkinson confunde una pregunta metodológica: cómo se identifica el efecto que los compañeros ejercen, con una afirmación teórica: si se hipotetiza que determinados efectos son atribuibles -aunque sea en parte, aunque sea mediados por otros factores- a los compañeros. 
y es para algunos aun una materia pendiente. Por ejemplo, en la tradición de investigación sobre la efectividad escolar, aunque el patrón general de resultados es favorable al argumento acerca de los efectos contextuales de la composición social de la escuela en los logros de sus alumnos, existe evidencia contradictoria que limitaciones metodológicas y teóricas han impedido resolver (Teddlie, Stringfield y Reynolds, 2000). Sólo investigaciones recientes que utilizan diseños metodológicos más adecuados contarían como evidencia sólida a favor de la presencia de un efecto de los compañeros estadísticamente significativo sobre los logros escolares, de magnitud relevante y positivo para los casos de contar con compañeros i) de mayor nivel socioeoconómico, ii) de mayor habilidad académica, iii) que no pertenecen a minorías étnicas o iv) que son mujeres (ver por ejemplo, Evans, Wallace, \& Schwab, 1992; Gaviria \& Raphael, 2001; Sacerdote, 2001; McEwan, 2003; Angrist \& Lang, 2004; Hoxby, 2000; Hoxby \& Weingarth, 2006; Schindler, 2003; Ding \& Lehrer, 2006; Duflo, 2008). La magnitud del efecto de los compañeros estaría también en relación inversa con la "distancia" de la unidad de agregación considerada respecto del proceso directo de enseñanza-aprendizaje (i.e. más fuerte a nivel del aula, más débil a nivel de la escuela) (Wilkinson et al., 2002). En síntesis: al menos para los resultados escolares, los compañeros sí importan.

Por último, existe en la literatura un fuerte debate acerca de dos aspectos cruciales referidos a la naturaleza del efecto de los compañeros: si este es constante o decreciente y si afecta de forma homogénea o diferenciada a estudiantes de diferentes características (Ammermueller \& Pischke, 2009; Hoxby \& Weingarth, 2006). La primera pregunta remite a la forma lineal o no lineal que adoptaría el efecto de los compañeros, es decir, si la acumulación de un determinado recurso positivo (e.g. compañeros académicamente hábiles) comienza o no sobre cierto límite (y cuál sería dicho límite) a presentar rendimientos decrecientes, ${ }^{2}$ o bien si es necesario cierto mínimo de acumulación (y cuál sería este) para que el efecto de los compañeros comience a manifestarse. La segunda pregunta busca determinar si todos los tipos de alumnos son igualmente sensibles al efecto de sus compañeros y en caso de no serlos, cuáles grupos se benefician o perjudican más significativamente en presencia de qué tipos de compañeros. La evidencia empírica acumulada hasta la fecha es insuficiente para responder este tipo de preguntas, las que -como veremos- son muy relevantes para el tema que nos ocupa.

\section{LA SEGREGACIÓN ESCOLAR}

Si los compañeros importan, entonces la pregunta acerca de cómo los alumnos son distribuidos entre las escuelas es también relevante. En su sentido más amplio, la segregación se refiere a la desigual distribución que poseen los diversos grupos sociales ya sea entre unidades de organización diferentes, entre zonas geográficas o en una combinación de ambos (James \& Taeuber, 1985), tal que dichas diferencias de distribución afectan las probabilidades de interacción entre miembros de los diferentes grupos sociales. Como

2 Por ejemplo, para el caso de Chile, McEwan (2003) encuentra que el efecto positivo de los compañeros en los logros de aprendizaje deja de aumentar alrededor de los 15 años de educación promedio de las madres del curso. 
se ve, la preocupación por la segregación social es en último término una pregunta por el grado en que miembros de diferentes grupos o categorías sociales pueden llegar a interactuar, compartir ciertas experiencias o ser afectados por condiciones compartidas.

Aunque el concepto de segregación es multidimensional, dos nociones han recibido mayor y especial atención en la literatura: la exposición y la similitud (Massey \& Denton, 1988; Gorard \& Taylor, 2002). La exposición se refiere a la probabilidad de interacción entre miembros de diferentes grupos o categorías sociales, es decir, el grado en que unos y otros están "expuestos" a personas de otros grupos; así, la segregación de un grupo se refiere al grado de aislamiento relativo de dicho grupo respecto a otras categorías sociales. La similitud se refiere al grado de desbalance con que los miembros de un grupo o categoría social se encuentran distribuidos entre diferentes unidades geográficas u organizacionales, de forma que dicho grupo se encontrará segregado si su distribución es muy desigual entre las unidades (ver Valenzuela, Bellei y De Los Ríos, 2009, para una discusión más detallada sobre las dimensiones empíricas del concepto de segregación).

Los estudios sobre la segregación deben por tanto definir dos cuestiones críticas: cómo se conforman los grupos o categorías sociales y cómo se delimita el espacio de convivencia potencial entre los miembros de diferentes grupos. Respecto de cómo definir grupos o subpoblaciones, la investigación sobre la segregación escolar-como hemos mencionado- ha abordado preferentemente las dimensiones raciales y de clase social, y en menor medida las dimensiones de género, étnicas y de habilidad académica. Nótese que, para ser relevante la definición de grupos "segregados", es indispensable contar con alguna hipótesis acerca de la ventaja relativa de un grupo sobre otro respecto de los logros educacionales. La cuestión del espacio es -en principio- mucho más simple en los estudios de segregación escolar que en los de segregación residencial, puesto que el aula y la escuela son los "espacios naturales" de la experiencia formativa en los sistemas educacionales (sin entrar por ahora en la discusión acerca de la importancia relativa del aula versus la escuela).

En términos empíricos, la investigación académica internacional ha documentado de modo sistemático que los sistemas educacionales tienden a segregarse de forma de producir un aislamiento relativo y un desbalance distributivo de las categorías sociales menos favorecidas, especialmente las minorías étnicas y raciales, y los alumnos de familias de clases sociales bajas. Muchos sistemas escolares también poseen mecanismos más o menos institucionalizados de agrupamiento de los alumnos según sus habilidades académicas, especialmente a nivel de la enseñanza secundaria. Ciertamente, los sistemas educacionales varían significativamente entre sí y en el tiempo respecto al grado de segregación de las diferentes categorías de estudiantes.

El hecho de que la distribución de los alumnos entre las diferentes escuelas no se produzca en forma aleatoria (único mecanismo que garantizaría la ausencia absoluta de segregación escolar) dirige la atención hacia la identificación de las causas que explican la ocurrencia de la segregación escolar. Aunque la investigación académica es menos sólida a este respecto, es posible afirmar que dichas causas son de múltiple naturaleza y que su efecto varía en el tiempo y entre países. Así, la segregación escolar es un fenómeno complejo causado por factores tanto internos como externos al campo educacional, los cuales se pueden agrupar en tres grandes dimensiones. Primero, factores contextuales (siendo la segregación residencial el más relevante); segundo, factores institucionales del propio sistema educacional (como, por ejemplo, la existencia de escuelas separadas por raza); y tercero, factores socio-culturales (como las preferencias de las familias y la 
existencia de escuelas "comunitarias" - de iglesias, colonias, o grupos de elite). Lo que hace especialmente difícil el estudio de la segregación escolar (así como las políticas para reducirla) es el hecho de que la segregación escolar es un fenómeno colectivo, una consecuencia agregada de acciones no necesariamente orientadas explícitamente a producirla (más aun, para muchos actores sociales la segregación puede ser una consecuencia indeseada y no anticipada de sus propias acciones).

Finalmente, la segregación escolar tendría -en principio- consecuencias educacionales en tres dimensiones diferentes. En primer término, empobrece la calidad de la experiencia formativa de los alumnos en un sentido amplio (convivencia social, educación ciudadana, habilidades transversales); en segundo lugar, disminuye los logros educacionales de los grupos vulnerables (en un sentido restringido: aumenta la deserción escolar y disminuye los aprendizajes académicos); finalmente, la segregación escolar dificulta el mejoramiento educacional y facilita la emergencia de fenómenos disfuncionales para el proceso de enseñanza-aprendizaje y la convivencia escolar. Lamentablemente, tampoco ha sido sencillo documentar empíricamente las consecuencias o efectos de la segregación escolar (en esto la literatura sobre segregación residencial lleva clara ventaja).

\section{SEGREGACIÓN Y EFECTO DE LOS COMPAÑEROS: MECANISMOS CAUSALES}

Uno de los desafíos importantes para el estudio de la segregación escolar es contar con una teoría que organice y fundamente los vínculos causales entre la condición de segregación y las consecuencias educacionales que a ella se atribuyen. Existen dos grandes hipótesis que pretenden explicar los mecanismos causales a través de los cuales opera la segregación escolar para producir ciertos grados de inequidad educacional: la noción de "capital social" (en la que no se profundizará en este trabajo) ${ }^{3}$ y la referida teoría del efecto de los compañeros.

La noción básica que vincula los conceptos de efecto de los compañeros y segregación escolar postula que, si se concentran en determinadas escuelas o salas de clase, alumnos de menores capacidades o recursos (en un sentido amplio), estos tendrán menos oportunidades de lograr aprendizajes, pues la mayor parte de sus compañeros presentará similares atributos o dificultades; esta desventaja adicional de los grupos de menores recursos en situación de segregación, opera tanto a nivel del contexto del aula-escuela como de los procesos de enseñanza-aprendizaje, ejerciendo efectos directos sobre los alumnos afectados e indirectos, especialmente por la vía de los docentes y los recursos disponibles en la escuela. Lo opuesto ocurrirá si se segrega a los alumnos con mayores recursos o capacidades. De esta forma, siguiendo con la terminología previamente establecida, la segregación escolar multiplicaría los "recursos facilitadores" para los alumnos no-vulnerables y los dividiría para los vulnerables; y realizaría la operación inversa con los "recursos obstaculizadores".

El capital social son las redes sociales y las relaciones de confianza que existen en una comunidad y que potencian la acción de las personas que a ella pertenecen. El capital social facilita la formación de los miembros jóvenes de la comunidad (es un recurso "mediador" entre el capital cultural de los adultos y la acumulación de capital humano por parte de los jóvenes), incluyendo el trabajo de la escuela (algunos casos tratados en la literatura son las escuelas públicas de una pequeña comunidad, las escuelas católicas, las "escuelas efectivas"). La hipótesis en este caso afirma que la segregación escolar multiplica el capital social de los grupos no-vulnerables y disminuye el capital social disponible para los grupos vulnerables. Los autores más relevantes de esta línea de pensamiento son P. Bourdieu, J. Coleman y R. Putnam. 
La teoría del efecto de los compañeros en educación no sólo provee una buena batería de hipótesis para explicar los mecanismos causales que vinculan la segregación escolar con los logros escolares; además, ilumina algunas de las preguntas más acuciantes sobre la segregación escolar desde el punto de vista de la equidad educacional y la productividad de los sistemas escolares.

Como se mencionó antes, no existe total claridad sobre si el efecto de los compañeros es homogéneo o heterogéneo entre categorías de alumnos, sin embargo, desde el punto de vista del logro académico de los alumnos, los estudios tienden a coincidir en que escuelas y aulas menos segregadas benefician comparativamente más a los alumnos más "vulnerables" (ya sea en términos económicos, raciales o académicos), quienes son asimismo más afectados negativamente por los contextos segregados. Mayor discrepancia ha existido en cambio en determinar si los alumnos "aventajados" son también beneficiados en contextos heterogéneos, si les son indiferentes (como sugieren Angrist \& Lang, 2004) o si son perjudicados (como sugieren Hoxby 2000 y Schindler, 2003).

De especial interés en este punto es la investigación acumulada sobre el efecto de agrupar a los alumnos -entre escuelas y al interior de ellas- según habilidades cognitivas o desempeño académico: según Mosteller, Light y Sachs (1996) la evidencia más robusta (aunque siempre escasa) tiende a favorecer la hipótesis de los efectos heterogéneos, en el sentido de que este tipo de "segregación" favorece a los alumnos de mayores habilidades, es indiferente o levemente negativa para los alumnos de habilidades intermedias y es negativa para los alumnos de menores habilidades. Gamoran (2000), Hallinan (1994), Oakes (1994) coinciden con esta conclusión; Slavin (1987, 1990) encuentra en cambio que la agrupación por alumnos según habilidad académica no tiene efectos positivos ni negativos relevantes; y Hattie (2002) considera sólo relevante -aunque pequeño -el beneficio positivo para los alumnos de mayores habilidades. ${ }^{4}$

Por otra parte, también se dijo que no hay certeza acerca de la forma funcional que adopta el efecto de los compañeros, es decir, si es o no lineal. Aunque la literatura sobre agrupamiento de estudiantes por habilidad sugiere que el efecto agregado total de la segregación sería cercano a cero (Hattie, 2002; Mosteller, Light y Sachs, 1996; Gamoran, 2000; Slavin, 1987, 1990), es decir, que la productividad total del sistema sería la misma con agrupamientos homogéneos o heterogéneos de alumnos según habilidad académica, la investigación sobre otras formas de segregación no es concluyente al respecto. Nótese que si el efecto de los compañeros tiene un rendimiento decreciente en el aprendizaje, la segregación escolar produciría una reducción en los logros educacionales a nivel agregado; en otras palabras, la segregación sería crecientemente ineficiente para el sistema escolar visto en su conjunto. Así, un efecto de los compañeros no lineal sugiere que es posible encontrar formas de distribución de los alumnos que son sistémicamente más productivas que otras, puesto que el cambio de un alumno a otra aula-curso no generaría un simple efecto de "suma-cero".

$4 \quad$ Es importante notar que casi todos los autores coinciden en señalar que la evidencia sobre los efectos de la segregación por habilidad cognitiva o desempeño académico es bastante poco consistente, lo que sugiere la relevancia de considerar las variables "mediadoras" entre dicha práctica y los logros de los alumnos, siendo la más importante de ellas la forma que adopta la pedagogía de los docentes. 
Establecido el campo intelectual en el cual se inserta la discusión académica sobre la segregación escolar, en las siguientes dos secciones se resume y analiza la investigación existente sobre la segregación socioeconómica y académica de la educación chilena. Como se verá, su estado de desarrollo es bastante más inicial que la literatura internacional discutida.

\section{SEGREGACIÓN SOCIOECONÓMICA DE LA EDUCACIÓN CHILENA}

La investigación científica se ha preocupado de estimar empíricamente el grado de segregación socioeconómica de la educación chilena. En un estudio reciente -utilizando el Índice de Disimilitud de Duncan (para una descripción metodológica del Índice de Duncan utilizado, ver Valenzuela, Bellei \& De Los Ríos, 2009)- se estimó que el grado de segregación socioeconómica de la población escolar chilena es relativamente alto, con valores del índice D cercanos o superiores a 0,5 para la población de menores recursos (también se encontró que al población escolar de mayores recursos está aun más segregada, con valores del índice D superiores a 0,6); además, se estimó que esta segregación ha tendido a aumentar en la última década y que es levemente mayor en la educación básica comparada con la educación media (Valenzuela, Bellei, De Los Ríos, 2010), tal como se aprecia en la Tabla 1.

Tabla 1. Segregación escolar a nivel nacional de los estudiantes de bajo nivel socioeconómico: Índice de Duncan: $30 \%$ de menor NSE

\begin{tabular}{|c|c|c|c|c|c|c|c|c|c|c|}
\hline & 1999 & 2000 & 2001 & 2002 & 2003 & 2004 & 2005 & 2006 & 2007 & 2008 \\
\hline $\begin{array}{c}4^{\circ} \\
\text { Básico }\end{array}$ & 0.51 & & & & & & 0.53 & 0.53 & 0.54 & 0.54 \\
\hline $\begin{array}{c}8^{\circ} \\
\text { Básico }\end{array}$ & & 0.50 & & & & & & & 0.53 & \\
\hline $\begin{array}{c}2^{\circ} \\
\text { Medio }\end{array}$ & & & 0.43 & & & & & 0.50 & & 0.50 \\
\hline
\end{tabular}

Fuente: Valenzuela, Bellei \& y De Los Ríos (2010).

El nivel de segregación socioeconómica de la población escolar chilena es comparativamente alto a nivel internacional. Valenzuela (2008), utilizando datos de la Prueba PISA-2006, estimó que Chile posee junto a Tailandia el más elevado índice D (estimado de modo comparable al referido en el párrafo anterior) de segregación de los alumnos pertenecientes al 30\% más pobre de la población escolar de entre los 57 países considerados, siendo los únicos países que superan el valor 0,50 en circunstancias que la mayoría de los países posee niveles de segregación escolar estimados usando el índice $\mathrm{D}$ con valores entre 0,3 y 0,4 . Lo mismo ocurre con la segregación de los alumnos con mayores recursos socioeconómicos: de la muestra de 57 países, Chile es el país donde el 30\% de alumnos de mayor nivel socioeconómico se encuentra más segregado, con valores incluso levemente superiores a 0,6. El Gráfico 1 muestra la distribución completa de ambos índices para los 57 países para los cuales se cuenta con información: en él 
queda claro que existe una estrecha relación entre la segregación de los más pobres y de los más ricos en una sociedad. Estos resultados son consistentes con los obtenidos por Willms (2010) y OECD (2010), aunque Elacqua (2009) sugiere que la segregación escolar chilena no sería tan elevada.

Gráfico 1. Segregación socioeconómica de la población escolar a nivel internacional: Índice de Duncan estimado para alumnos pertenecientes al 30\% más bajo y al 30\% más alto nivel socioeconómico (57 países incluidos en el gráfico).

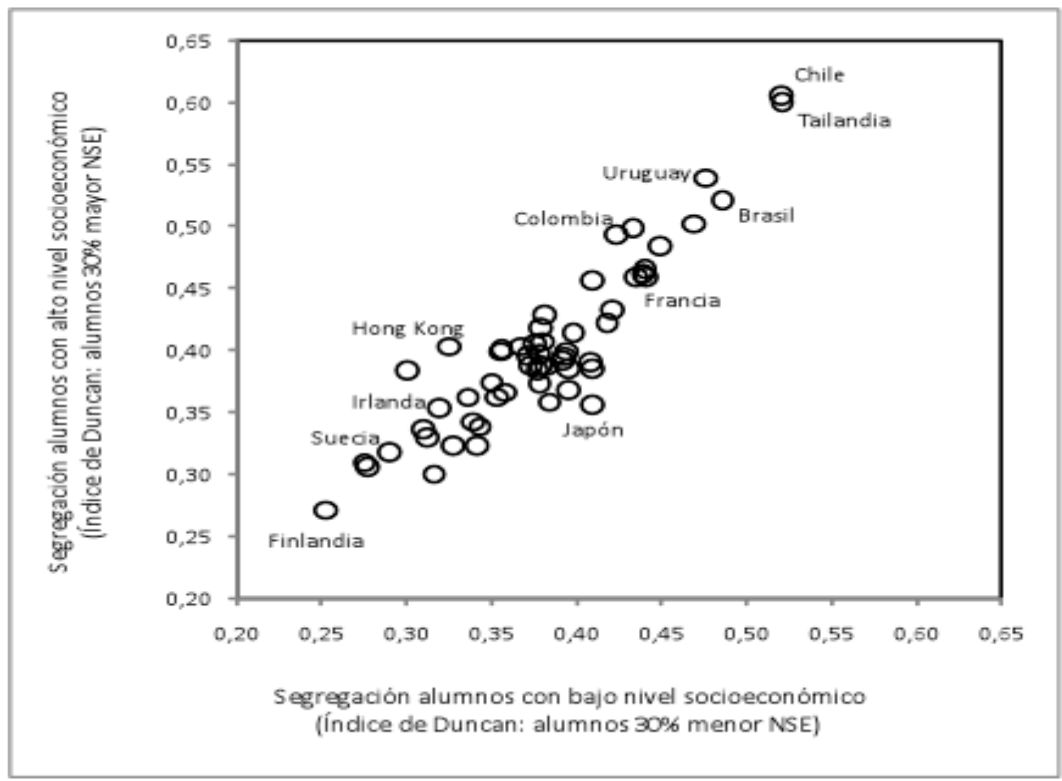

Fuente: elaboración propia en base a Valenzuela (2008).

Existen diversas hipótesis respecto a las causas o factores asociados a la producción de la segregación socioeconómica del sistema escolar chileno, referidas tanto a aspectos contextuales como institucionales y culturales, pero la mayor parte de éstas no han sido testeadas empíricamente.

Un estudio reciente (cuyo diseño permite testear la presencia de correlaciones parciales en diferentes contextos, pero no realizar inferencias causales) abordó esta pregunta, incluyendo factores de contexto e institucionales (ver Valenzuela, Bellei y De Los Ríos, 2009). Los hallazgos indican que la segregación socioeconómica de la población escolar chilena estaría fuerte y positivamente asociada con i) la segregación residencial de las comunas, ii) la presencia y relevancia de la educación privada no-subvencionada en la comuna, iii) la presencia y relevancia de la educación privada subvencionada en la comuna, y iv) la relevancia del financiamiento compartido en la comuna (sistema de co-pago de las familias en el sector de escuelas privadas subvencionadas).

Es decir, la evidencia disponible confirma que la segregación escolar está fuertemente afectada por la segregación residencial, pero que en ningún caso se reduce a ella, sino que algunos dispositivos internos del sistema escolar contribuyen también a producirla. 
En particular, en el caso chileno, dos elementos parecen claves: el rol que juegan las escuelas privadas y el efecto discriminador que ejerce el co-pago vía financiamiento compartido. En efecto, el mencionado estudio también encontró que la segregación escolar de los alumnos socioeconómicamente más vulnerables es mayor en los establecimientos privados (aunque sean subvencionados) que en los públicos (Valenzuela, Bellei y De Los Ríos, 2009), hallazgo replicado luego por Elacqua (2009). Más aun, al interior de los establecimientos privados subvencionados por el estado, la segregación escolar sería mayor en aquellos que cobran a las familias, comparados con los establecimientos gratuitos (Elacqua, 2009).

Los efectos educacionales de la segregación socioeconómica del sistema escolar chileno están aún por estudiarse. Ciertamente, se encuentra muy documentado el efecto que el nivel socioeconómico de los padres tiene sobre el resultado escolar de sus hijos, pero los académicos han discrepado sensiblemente sobre la relevancia y la forma de incluir en sus estudios el potencial efecto que el nivel socioeconómico de los padres agregado a nivel de la escuela o la sala de clases tendría sobre los resultados escolares de los alumnos (i.e. no de "sus" hijos). Una revisión bibliográfica y metodológica de este asunto se encuentra en Bellei (2009), donde además se demuestra que dichos efectos de composición (medida aproximada del "efecto de los compañeros") son importantes y significativos en los resultados de aprendizaje de los alumnos, más allá del efecto "directo" de las características de su propia familia. Este hallazgo ha sido replicado en el marco de un estudio comparativo con España, Polonia y Uruguay, donde se encontró que para Chile la magnitud de este "efecto de los compañeros" era mayor tanto para los resultados de Lectura (Bellei, Valenzuela, Osses y Sevilla, 2009), como de Matemáticas (Valenzuela, Bellei, Osses y Sevilla, 2009), especialmente al comparársele con los países europeos. De hecho, un reciente estudio de UNESCO muestra que los efectos de composición sobre los resultado de aprendizaje serían en general elevados en los países de América Latina (Treviño et al., 2010).

\section{SEGREGACIÓN ACADÉMICA DE LA EDUCACIÓN CHILENA}

La segregación académica ha sido menos estudiada en Chile que la segregación socioeconómica. Existen varios trabajos que hacen referencia a ella, pero de modo más bien indirecto y sin que se cuente a la fecha con investigaciones empíricas rigurosas acerca de su magnitud, causas y consecuencias. Se trata sin duda de un campo por explorar. Una dificultad adicional para este tipo de estudios, comparados con los de segregación socioeconómica, es que simplemente observar la distribución de alumnos según logros escolares no proporciona una estimación rigurosa de la segregación, puesto que en dichos logros se combinan los efectos de los múltiples mecanismos que distribuyen desbalanceadamente a los alumnos (segregación) con los diferentes niveles de efectividad escolar de los establecimientos (productividad) -se vuelve sobre este punto en la sección final.

En términos de la incidencia de la segregación académica de los alumnos del 30\% de mejor desempeño escolar, Guinguis (2008) -utilizando una metodología análoga a la de Valenzuela, Bellei y De Los Ríos (2009)- estima para el caso de Matemáticas un índice Duncan para cuarto básico de 0,37 en 1999 y 0,39 en 2006, y un índice Duncan para segundo medio de 0,50 en 2001 y 0,53 en 2006. De acuerdo a estos resultados, la 
segregación académica sería intermedia en la enseñanza básica y muy alta en la educación media, con una leve tendencia a incrementarse en ambos niveles. Es interesante notar que para Lenguaje las estimaciones son menores, especialmente en segundo medio (en 2006, 0,36 y 0,44 para cuarto básico y segundo medio, respectivamente), sin que se aprecie una tendencia al aumento en el tiempo (Guinguis, 2008). Este hallazgo podría indicar que los establecimientos educacionales dan más relevancia al desempeño de los alumnos en Matemáticas al momento de tomar de decisiones de admisión, expulsión y agrupamiento de los alumnos.

Una medida indirecta de segregación académica que ha sido utilizada por la literatura es estimar la proporción de la variación de resultados escolares que se da "entre" los establecimientos en comparación con la variación que se da "al interior de" los establecimientos. La lógica es que en un sistema escolar en que los alumnos fuesen distribuidos al azar, toda la variación de habilidades académicas iniciales se daría "al interior de" los establecimientos, quedando la variación "entre" establecimientos reducida prácticamente a cero; así, mientras mayor sea la proporción de la variación de resultados "entre" establecimientos mayor sería el grado en que los alumnos se encuentran académicamente "segregados" en un sistema escolar. Ramírez (2007), analizando datos del SIMCE-19995 de cuarto básico en Matemáticas, encuentra que un $27 \%$ de la varianza de puntajes se da "entre" escuelas, ${ }^{6}$ proporción que considera similar a la reportada para otros países. Bellei (2009), usando datos del SIMCE 2003 de segundo medio, estima que aproximadamente la mitad de la variación de los puntajes en Matemáticas y poco más de un tercio en Lenguaje se da "entre" establecimientos, lo cual considera una fracción elevada. Según datos de la prueba PISA, Chile ocupa el lugar 10 entre 57 países con mayor varianza de resultados entre establecimientos en Ciencias-2006 (OECD, 2007) y el lugar 18 entre 65 países en Lectura-2009 (OECD, 2010). Sin embargo, es importante, a mi juicio, no sólo observar los valores absolutos de la varianza "entre" e "intra" escuelas sino la relación entre ambos indicadores; así, mientras en el promedio de países de la OECD la varianza "intra" escolar de resultados de Lectura-2009 es 1,55 veces mayor que la varianza "entre" escuelas, en Chile esta relación es 0,82 , es decir, la varianza "entre" escuelas supera a la varianza "intra" escuela (OECD, 2010).

Como se mencionó, el problema de las estimaciones anteriores es que no permiten distinguir entre segregación académica y diferencias de productividad escolar; para ello, sería necesario conocer las causas que derivan en la distribución desbalanceada de los alumnos con diferente nivel de rendimiento. En Chile solo existe información indirecta acerca de los mecanismos de segregación académica entre escuelas.

Uno de dichos dispositivos son los procesos de admisión de alumnos que en muchos establecimientos incluyen la aplicación de diferentes instrumentos de selección de postulantes en base a su potencial académico. Por ejemplo, Gauri (1998) en una encuesta realizada en 1993-1994 en la región metropolitana de Santiago, encontró que el 82\% de los alumnos de escuelas privadas no subvencionadas, el $37 \%$ de los alumnos de escuelas

\footnotetext{
5 SIMCE es el Sistema de Medición de la Calidad de la Enseñanza en Chile, que consiste en una batería de tests en Lenguaje y Matemáticas (complementados a veces con Ciencias) aplicados anualmente a todos los alumnos de cuarto grado, y bi-anualmente a los alumnos de octavo y décimo grado.

6 La autora distingue adicionalmente los niveles regional, provincial y comunal, pero aquí todos ellos los consideramos parte de la varianza "entre" escuelas.
} 
privadas subvencionadas y el $18 \%$ de los alumnos de escuelas municipales había tenido que rendir algún tipo de test de selección para ser admitido en su establecimiento; para Gauri, las escuelas con mejores resultados obtienen parte significativa de su ventaja por este medio. Bellei (2009), usando datos del SIMCE-2003 de segundo medio, encontró que el $85 \%$ de los padres de establecimientos privados no subvencionados, el $73 \%$ de los padres de establecimientos privados subvencionados y el 59\% de los padres de establecimientos municipales reportaron que sus hijos debieron rendir dichos test de admisión. Ciertamente, la selección académica se complementa con la discriminación por precio: Roco (2010) muestra que la presencia de la selección académica es el doble entre las escuelas particulares subvencionadas que cobran a las familias en comparación con aquellas que son gratuitas (dos tercios versus un tercio de cobertura, respectivamente); sin embargo, ambos fenómenos no son reductibles entre sí, como lo demuestra empíricamente Bellei (2009): la selección académica parece operar como un "segundo filtro" una vez superada la barrera económica.

Un dispositivo adicional de segregación académica es la expulsión de alumnos que presentan menor rendimiento o tienen características asociadas a él (como problemas de disciplina). Este fenómeno no se encuentra bien documentado en Chile. De acuerdo a datos del SIMCE-2003 de segundo medio, la mayoría de los padres de establecimientos particulares sin subvención, uno de cada tres padres de establecimientos particulares subvencionados y uno de cada siete padres de establecimientos municipales, afirma que en su establecimiento se expulsa a los alumnos que repiten curso. Según los directores de los establecimientos chilenos participantes en la prueba PISA-2009, en el 30\% de los establecimientos es una práctica establecida expulsar a los alumnos de bajo rendimiento y en el $75 \%$ lo es expulsar a los alumnos con problemas disciplinarios (OECD, 2010). Estas prácticas de selección "durante el proceso" de escolarización también explican una parte significativa de la ventaja que obtienen los establecimientos de mejor rendimiento académico (Bellei, 2009).

La segregación académica es ciertamente un fenómeno multicausado, cuyos antecedentes exceden el contexto inmediato de la escuela, donde se aplican los mencionados procesos de admisión-expulsión de alumnos. En términos del contexto social-institucional, ya se ha dicho que la segregación socioeconómica de los estudiantes produce indirectamente segregación académica; adicionalmente, características institucionales y curriculares del sistema escolar chileno también refuerzan la segregación académica, como, por ejemplo, la división en el noveno grado de escolarización entre educación general pre-universitaria y educación técnica vocacional, o la existencia de establecimientos "de excelencia académica" especializados en la formación de la elite. Finalmente, factores socio-culturales combinados con características institucionales y políticas educacionales también contribuirían: si los padres tienen libertad para elegir la escuela en que matriculan a sus hijos, si las autoridades publican y enfatizan el uso de indicadores de logro escolar en la elección (e.g. el SIMCE) y los padres obtienen y valoran dicha información, se genera desde la demanda un reforzamiento de la segregación académica. Nótese que la evidencia empírica no es muy sólida a este respecto por cuanto una fracción menor de los padres manifiesta usar el puntaje SIMCE para elegir donde matricular a sus hijos (Elacqua y Fábrega, 2006), sin embargo, otros indicadores "indirectos" de logro escolar más usados por los padres -como la calidad percibida, el prestigio de la escuela o el "tipo de alumnos" que a ella asisten- contribuyen también a la segregación académica.

En términos de las consecuencias de la segregación académica en los logros escolares, sólo existen investigaciones empíricas que han realizado mediciones indirectas. Por 
ejemplo, usando datos del SIMCE-2003 en Matemáticas, Bellei (2009) estimó un efecto promedio positivo de asistir a un establecimiento en que la totalidad de los alumnos hayan sido seleccionados mediante la aplicación de tests de admisión equivalente a 0,53 desviaciones estándar, efecto de tamaño grande para este tipo de estudios. Más aun, dichas estimaciones sugirieron que los alumnos de enseñanza básica que han cambiado de una escuela a otra son más sensibles al efecto positivo de asistir a un establecimiento académicamente selectivo y al efecto negativo de asistir a uno no selectivo (Bellei, 2009). Este último resultado es importante porque muestra cómo la segregación académica es un proceso que parece irse refinando conforme los alumnos avanzan en su carrera escolar, lo que podría estar asociado con los resultados de Guinguis (2008) antes discutidos.

Finalmente, aunque la segregación académica y la segregación socioeconómica apuntan a dimensiones diferentes de la organización y funcionamiento de los sistemas escolares, ambos fenómenos -como se argumentó- tienden a estar fuertemente relacionados en la práctica. Una imagen de dicha asociación empírica se obtiene del gráfico 2 , referido a los niveles de "inclusión" social y académica de las escuelas en 64 países: en general (aunque existen interesantes excepciones como Tailandia y Holanda que demuestran que ambos aspectos son separables), a mayor inclusión social, mayor inclusión académica. Como se ve, Chile sigue este patrón de un modo casi paradigmático, puesto que es uno de los países con menor inclusión social en sus escuelas y simultáneamente posee una baja inclusión académica, situación que lo enfrenta con -por ejemplo- Finlandia, país con altísimos grados de inclusión en ambas dimensiones.

Gráfico 2. Inclusión social e inclusión académica (64 países incluidos en el gráfico, índice estandarizado 0-100 para países participantes en PISA-2009 de Lectura).

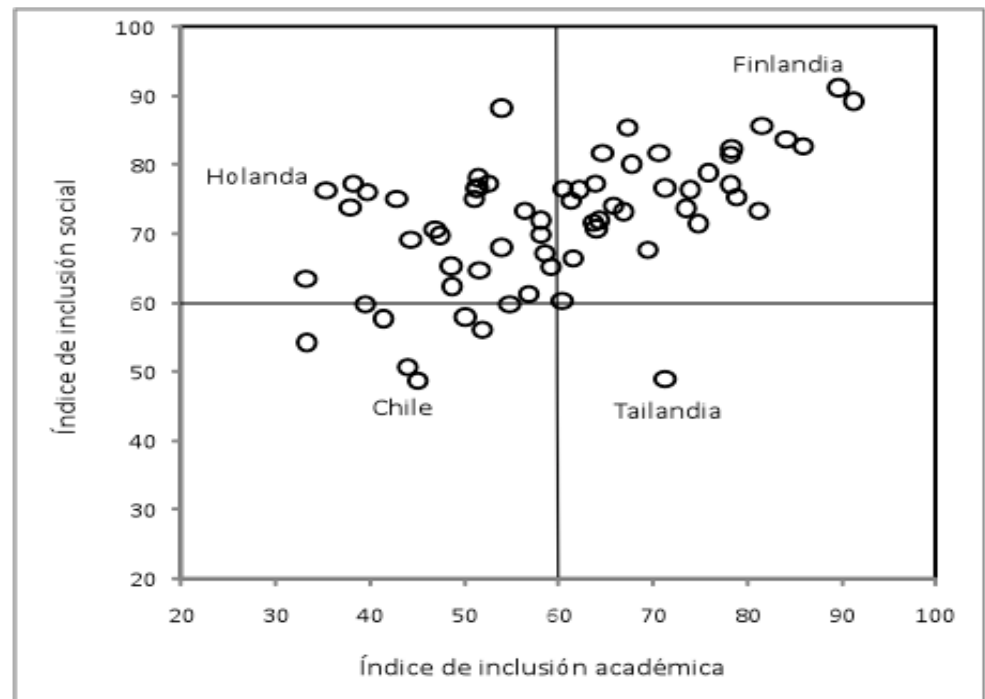

Fuente: elaboración propia en base a datos PISA-2009 (OECD, 2010).

Nota: los índices representan el porcentaje de la varianza total que corresponde a la varianza "intra escuela" tanto en los resultados PISA-Lectura como en el índice de nivel socioeconómico y cultural de los alumnos. Valores mayores en estos índices indican escuelas internamente más heterogéneas, valores menores indican escuelas internamente más homogéneas en la dimensión correspondiente. 


\section{CONCLUSIONES Y PERSPECTIVAS PARA LA INVESTIGACIÓN}

\subsection{CONCLUSIONES}

En este trabajo se ha hecho una revisión acerca de la segregación escolar en Chile, situándola en la perspectiva de la literatura internacional. El estudio de la segregación del sistema educacional chileno tiene especial interés dada su fuerte orientación de mercado: financiamiento tipo voucher, copago de las familias, libre elección escolar, competencia abierta entre escuelas privadas y públicas, uso intensivo de tests a escala nacional, etc. Buena parte del debate en los países que se encuentran considerando la aplicación de este tipo de políticas se ha centrado precisamente en sus potenciales efectos sobre la equidad, incluida la segregación, por lo que un mayor conocimiento del caso chileno podría informar dicho debate.

El argumento principal aquí desarrollado es que la segregación socioeconómica y la segregación académica de la población escolar no son rasgos anecdóticos de los sistemas escolares, sino que constituyen factores relevantes de la (in)equidad educativa, en tanto afectan diferencialmente las oportunidades y logros de aprendizaje de los alumnos. De las diferentes teorías que intentan explicar cómo se producirían dichos efectos, se ha argumentado sobre el valor del concepto de "efecto de los compañeros": la segregación modularía la probabilidad de los diferentes grupos de estudiantes de ser afectados (positiva o negativamente) por sus compañeros. Finalmente, se ha afirmado que los efectos de la segregación se producen tanto directamente sobre los alumnos y sus procesos de enseñanza-aprendizaje, como indirectamente a través del ambiente escolar y los profesores. La articulación teórica y la indagación empírica de los conceptos de segregación escolar y efecto de los compañeros constituyen a mi juicio un terreno fértil para los avances de la Sociología de la Educación y las demás Ciencias Sociales.

Para el caso chileno, los resultados de los estudios empíricos analizados muestran que la segregación académica y sobre todo la segregación socioeconómica de los estudiantes, en educación básica y media, son comparativamente altas (siendo más elevadas en las escuelas privadas que en las públicas). La segregación socioeconómica parece haber aumentado en la última década en Chile; mientras la segregación académica parece incrementarse (especialmente para Matemáticas) al pasar de la enseñanza básica a la media.

La escasa investigación existente sobre las causas de estos fenómenos muestra que la segregación socioeconómica está vinculada a la segregación residencial, pero está lejos de ser su simple reflejo; otros factores, propios del sistema educacional, contribuyen significativamente a su producción, en especial las dinámicas de mercado de la educación chilena, expresadas en la fuerte presencia de escuelas privadas con y sin subvención estatal, y el sistema de copago de la educación escolar subvencionada. A su vez, la evidencia disponible indica que la segregación académica se asocia a la segregación socioeconómica, pero no se reduce a ella: las prácticas de selección de los estudiantes en los procesos de admisión y durante la carrera escolar (especialmente en las escuelas privadas), así como las dinámicas de autoselección de las familias, también contribuirían a producirla.

Aunque no existen estudios en Chile sobre las consecuencias de la segregación escolar tanto socioeconómica como académica (en términos de las oportunidades y resultados educacionales de los estudiantes), evidencia indirecta sugiere que ambos tipos de segregación están fuertemente asociados a los logros académicos de los alumnos medidos 
por pruebas estandarizadas, en el sentido de favorecer a quienes asisten a escuelas cuyo alumnado es de mayor nivel socioeconómico y/o de mejor desempeño académico.

Finalmente, no ha sido el propósito de este trabajo abordar los desafíos que la segregación escolar presenta a la política educacional, pero es conveniente una breve reflexión al respecto: si el argumento desarrollado -que vincula la segregación con la equidad y la productividad del sistema escolar- es correcto, entonces ambos tipos segregación son materias relevantes para la política educacional; máxime si ambos fenómenos son en cierta medida un producto de instituciones, políticas y prácticas del propio sistema educacional. Los tomadores de decisiones deberían al menos identificar y evitar las políticas que -como la imposición de aranceles a las familias- agudizan la segregación socioeconómica. Asimismo, sería indispensable revisar la justificación educacional de los mecanismos de segregación académica, puesto que sabemos que -dejados a su propia dinámica- estos tienden a reforzar y a aumentar la inequidad educacional. Ciertamente, si nuestro razonamiento sobre las ventajas relativas que algunos grupos de estudiantes obtienen con la segregación escolar es correcto, autoridades y educadores deberían también estar conscientes de que es altamente probable que las políticas destinadas a contener o disminuir la segregación enfrenten fuertes resistencias en el sistema educacional y en la sociedad más amplia, haciéndonos así recordar que antes que un campo de investigación académica, la educación es un campo de lucha social.

\subsection{PERSPECTIVAS PARA LA INVESTIGACIÓN SOBRE SEGREGACIÓN ESCOLAR EN CHILE}

Desafortunadamente, una conclusión adicional de este trabajo es que existe poca investigación académica sobre la segregación escolar en Chile, a pesar de que ha habido creciente debate público e iniciativas políticas al respecto. Para contribuir a superar esta situación, a continuación se esbozará algunos aspectos de la segregación en Chile cuyo estudio es particularmente relevante, haciendo además consideraciones metodológicas importantes de tener en cuenta para futuras investigaciones.

Respecto de la segregación socioeconómica, trabajos recientes han comenzado a llenar el vacío que existía sobre su magnitud y evolución, sin embargo, el estudio de sus causas y consecuencias está prácticamente por iniciarse. Estudiar las causas de la segregación socioeconómica de la población escolar en Chile enfrenta una dificultad importante: la información histórica con que se cuenta al respecto es muy limitada, por lo que la evolución que es posible observar no siempre produce la variabilidad requerida para conocer sus causas; esto es especialmente complicado dado el alto nivel de segregación observado actualmente: ¿fue siempre tan segregado el sistema escolar chileno? ¿cuánto de su segregación se puede atribuir a la implantación del modelo de mercado a inicios de 1980? Será muy difícil responder empíricamente este tipo de preguntas. En consecuencia, un área particularmente fructífera de investigación sería, por una parte, diseñar "cuasi experimentos" que comparen transversalmente diferentes realidades geográficas y logren ahí identificar la incidencia de factores relevantes, y -por otra- identificar "experimentos naturales" que permitan aislar el efecto de variables significativas. Algunas políticas educacionales recientemente inauguradas (como la creación de un voucher socialmente diferenciado) ofrecen buenas oportunidades a este respecto.

Se sabe muy poco sobre las consecuencias de la segregación socioeconómica de la población escolar chilena. Avanzar en este frente no sólo tiene un interés académico: 
muchos educadores y tomadores de decisión del campo educacional sólo están dispuestos a asignar relevancia al "problema" de la segregación si se demuestra empíricamente sus hipotetizados efectos negativos. Dados los sesgos de selección presentes y el carácter circular o bidireccional de muchos de los potenciales efectos, vincular causalmente la segregación con los resultados escolares de los alumnos es enormemente difícil. Ciertamente, algunos estudios citados en el texto proveen buenos ejemplos de diseños altamente sofisticados que han avanzado en esa monumental tarea. Sin embargo, este es un campo en el cual, a mi juicio, se debe evitar el reduccionismo metodológico en dos importantes sentidos. Primero, por mucho que refinen sus estimaciones, los estudios cuantitativos seguirán siendo limitados para conocer cómo opera la relación causal entre segregación, oportunidades y logros escolares de los alumnos: este es un ámbito privilegiado para el desarrollo de estudios cualitativos, capaces de mostrar cómo las experiencias formativas de los alumnos son afectadas por el entorno segregado en que se desenvuelven. Segundo, las consecuencias relevantes de estudiarse no son sólo aquellas medidas por los tests estandarizados de resultados de aprendizaje: la preocupación por la segregación escolar apunta también -a nivel del estudiante- hacia los aprendizajes en otras áreas del currículum, la adquisición de habilidades de diferente tipo y el desarrollo de actitudes y valoraciones sociales, y -a nivel agregado- hacia la capacidad de mejoramiento escolar, el aumento de la calidad y equidad sistémicas y una sana convivencia escolar.

Respecto de la segregación académica de los alumnos el terreno está aún más virgen. En primer lugar, es preciso desarrollar metodologías e indicadores que midan de un modo más directo el fenómeno de la segregación y permitan diferenciarlo más claramente de las consecuencias causadas por la distinta efectividad de las escuelas y docentes: a diferencia de la condición socioeconómica del estudiante, su rendimiento académico puede ser directamente afectado por la acción del docente o la escuela a la que asiste. Ciertamente, se podría argumentar que dichas diferencias de efectividad son parte de la segregación académica, pero a mi juicio ese argumento confunde dos fenómenos de naturaleza distinta: si en un sistema dado los alumnos fuesen distribuidos al azar entre las escuelas y no pudiesen ser luego transferidos entre ellas, la variación de resultados observada luego "entre" escuelas sería una medida de efectividad diferencial de las escuelas, no de segregación académica. La segregación académica refiere a la distribución desbalanceada de estudiantes de diferente nivel de habilidad o desempeño. La complicación empírica surge de dos fuentes principales: la primera es que pocas veces se cuenta con una medida de la habilidad o desempeño de los estudiantes que no haya sido afectada por la escuela a la que asiste; la segunda es que la efectividad demostrada por las escuelas afecta a las familias que eligen enviar a sus hijos a ella y -frecuentemente, especialmente en el caso chileno- a las políticas de admisión y el nivel de selectividad de las escuelas. Como estos fenómenos evolucionan en el tiempo, cuando el investigador recoge sus datos en un momento dado, en verdad recoge de algún modo el acumulado histórico de la escuela en estas dimensiones, todo lo cual "sesga" su estimación del fenómeno de la segregación académica.

Lo anterior hace aún más relevantes los estudios destinados a identificar las causas o los factores asociados a la segregación académica: paradojalmente, en este caso, tal parece que sería más productivo avanzar en el conocimiento de las causas de la segregación que en la medición precisa de su magnitud. En efecto, a mi juicio, conociendo rigurosamente cómo operan los múltiples mecanismos de segregación académica, será posible filtrarla 
más refinadamente de las mencionadas diferencias de productividad escolar. También aquí se requiere una combinación de estudios cuantitativos que dimensionen la incidencia real de dichos mecanismos, con estudios cualitativos que profundicen en el modo en que estos operan en la práctica (más allá de las declaraciones formales o de los reportes de actores muchas veces interesados). ¿Cuánto los procesos de selección de alumnos son efectivamente basados en el desempeño potencial de estos? ¿cómo se combinan estos procesos de selección académica con aquellos otros de selección social de la matrícula? ¿cómo ocurren en la práctica los procesos de transferencia de alumnos? ¿cómo se toman las decisiones de matrícula al pasar de la enseñanza primaria a la secundaria? Sabemos sorprendentemente poco de cuestiones críticas para el funcionamiento del sistema escolar chileno.

El estudio de los efectos de la segregación académica enfrenta los mismos desafíos metodológicos que los mencionados a propósito de la segregación socioeconómica, pero en este caso de un modo aún más complejo de investigar e interpretar. En efecto, el hecho de haber analizado conjuntamente en este artículo ambos tipos de segregación no debe confundir: ellas poseen valoraciones educacionales profundamente diferentes. La segregación socioeconómica es difícilmente defendible desde un punto de vista educacional, quienes la aceptan lo hacen más bien porque piensan que es muy difícil modificarla o porque creen que no tiene mayor relevancia, pero -hasta donde conozco- no se han elaborado argumentos que defiendan el valor educacionalmente positivo de este tipo de segregación. La segregación académica, en cambio, aunque no es universalmente reconocida, posee un fuerte arraigo en los sistemas escolares (al punto que se encuentra institucionalizada en diferentes formas) y es respaldada por diversos argumentos que defienden sus bondades desde el punto de vista de la eficacia educacional. Muchos educadores sostienen que el agrupamiento de alumnos con similar rendimiento o capacidad permite un ajuste más fino de la pedagogía a las necesidades de los estudiantes, de forma que produciría mayores oportunidades de aprendizaje, siendo potencialmente más efectivo que el agrupamiento heterogéneo de alumnos. El que la evidencia empírica no respalde demasiado este argumento es interpretado por ellos como un requerimiento para preparar mejor a los docentes con miras a hacer más productivo este arreglo, no como una razón para desecharlo. En consecuencia, un área fructífera a este respecto es el estudio de situaciones de muy alta segregación académica, socialmente aceptadas y educacionalmente consolidadas, como son -en un extremo- los establecimientos públicos de elite (e.g. liceos tradicionales) y -en el otro extremo- las clases remediales para alumnos con rezago escolar. Estos contextos probablemente sean los "laboratorios" que muestren el máximo potencial de este tipo de prácticas. También es relevante estudiar hasta qué punto la presencia masiva de procesos de segregación académica pueda estar afectando negativamente la productividad de la educación chilena, por la vía, por ejemplo, de producir lo que se podría denominar "competencia espuria", dado que las escuelas alteran sus indicadores de efectividad al seleccionar estudiantes con mayor potencial académico, en un contexto de libre oferta y demanda escolar, informada por test estandarizados. En definitiva, la investigación académica sobre la segregación escolar en Chile recién comienza. 


\section{REFERENCIAS BIBLIOGRÁFICAS}

Ammermueller, A., \& Pischke, J. (2009). Peer Effects in European Primary Schools: Evidence from PIRLS. Journal of Labor Economics, n. 27, 315-348

Ananat, E. \& Washington, E. (2009). "Segregation and Black Political Efficacy." Journal of Public Economics, vol. 5-6, 807-822.

Ananat, E. (2007). "The Wrong Side(s) of the Tracks: The Effects of Racial Segregation on City Outcomes". NBER Working Paper 13343. En línea; disponible en: http://www.nber.org/papers/ w13343

Angrist, J.D. \& Lang, K. (2004). "Does School Integration Generate Peer Effects? Evidence from Boston's Metco Program.” American Economic Review, American Economic Association, vol. 94, n. $5,1613-1634$.

Bellei, C. (2009). "The Private-Public School Controversy: The Case of Chile". P. Peterson y R. Chakrabarti (Eds.), School Choice International. Harvard: Harvard University Press.

Bellei, C., J. P. Valenzuela, A. Osses y A. Sevilla. (2009). "¿Qué explica las diferencias de resultados PISA Lectura entre Chile y algunos países de la OCDE y América Latina?”. En ¿¿Qué nos dice PISA sobre la educación de los jóvenes en Chile? (pp. 149-170). Santiago: Mineduc - OEI.

Cutler, D., \& Glaeser, E. (1997). Are Ghettos Good or Bad? Quarterly Journal of Economics, $n$. $112,827-872$.

Ding, W., \& Lehrer, S. (2006). Do Peers Affect Student Achievement in China's Secondary Schools? NBER Working Paper 12305.

Duflo, E; Dupas, P. y Kremer, M. (2008). Peer Effects, Pupil-Teacher Ratios, and Teacher Incentives: Evidence from a Randomized Evaluation in Kenya. NBER Working Paper 14475.

Duncan, O., \& Duncan, B. (1955). A methodological analysis of segregation indexes. American Sociological Review, n. 20, 210-217.

Elacqua, G. (2009). The impact of school choice and public policy on segregation: Evidence from Chile. Documento de Trabajo n. 10. Santiago de Chile: Centro de Políticas Comparadas de Educación, Universidad Diego Portales.

Elacqua, G. y R. Fábrega (2006). "El consumidor de la educación: el actor olvidado de la libre elección de escuelas en Chile". En S. Cueto (Ed.), Educación y brechas de equidad en América Latina. Santiago de Chile: PREAL.

Evans, W., Wallace, O., \& Schwab, R. (1992). Measuring Peer Group Effects: A study of Teenage Behavior. Journal of Political Economy, n. 100, vol. 5, 84-117.

Gamoran, A. (2000). Is ability grouping equitable? En R. Arum e I. Beattie (Eds.), The structure of schooling. McGraw-Hill.

Gauri, Varum. (1998). School Choice in Chile. Two decades of educational reform. Pittsburgh: Pittsburgh University Press.

Gaviria, A., \& Raphael, S. (2001). School Based Peer Effects and Juvenile Behavior. Peer Review of Economics and Statistics, vol. 83, n. 2, 257-268.

Glaeser, E., \& Vidgor, J. (2001). Racial Segregation in the 2000. Census: promising news. Center on Urban \& Metropolitana Policy, Survey Series, 1-18.

Guinguis, S. (2008). Segregación de las elites en el sistema escolar chileno. Memoria de título Ingeniero Comercial, Escuela de Economía y Administración. Santiago: Universidad de Chile.

Hallinan, M. (1994). Traking: from theory to practices (The traking debate). Sociology of Education, vol. 67, n. 2, 79-91.

Hattie, J. (2002). Classroom composition and peer effects. International Journal of Educational Research, n. 37, 449-481.

Hoxby, C. (2000). Peer Effects in the Classroom: Learning from Gender and Race Variation. NBER Working Paper, n. 7867. En línea; disponible en: http://www.nber.org/papers/w7867. pdf?new_window $=1$ 
Hoxby, C., \& Weingarth, G. (2006). Taking Race Out of the Equation: School Reassignment and the structure of peer effects. Mimeo. Cambridge, MA: Harvard University.

Jenkins, S.; Micklewright, S. \& Schnepf, S. (2006). Social Segregation in Secondary Schools: How does England Compare with Other Countries? Southampton, GB: University of Southampton, Southampton Statistical Sciences Research Institute \& Policy Working. Paper A06/01, January.

McEwan, Patrick (2003). Peer effects on student achievement: evidence from Chile. Economics of Education Review, n. 22, 131-141.

Mosteller, F; R. Light \& J. Sachs (1996). Sustained inquiry in education: lessons from skill grouping and class size. Harvard Educational Review, vol. 66, n. 4, 797-842.

Oakes, J. (1994). Traking: from theory to practices (The traking debate). Sociology of Education, vol. $67, n .2,79-91$.

OECD (2007). Informe PISA 2006: Competencias científicas para el mundo del mañana. Paris: OECD, Santillana.

OECD (2010). PISA 2009 Results: Overcoming Social Background - Equity in Learning Opportunities and Outcomes (Volume II). Paris: OECD.

OECD (2010). PISA 2009 Results: What Makes a School Successful? - Resources, Policies and Practices (Volume IV). Paris: OECD.

Orfield, G. (2001). Schools more separate: Consequences of a decade of resegregation. Cambridge, MA: The Civil Rights Project, Harvard University.

Ramírez, M.J. (2007). Diferencias dentro de las alas de clases: distribución del rendimiento en Matemáticas. Estudios Públicos, n. 106, 5-22.

Rist, R. C. (2000). Student social class and teacher expectations: The self-fulfilling prophecy in ghetto education. Harvard Educational Review, vol. 70, n. 3, 257-301

Roco, R. (2010). Caracterización de los establecimientos educacionales en Chile: la necesidad de nuevas consideraciones. Ponencia presentada al Primer Congreso Interdisciplinario de Investigación en Educación, CIAE, Universidad de Chile y CEPPE, Pontificia Universidad Católica de Chile

Rosenthal, R. (1987). Pygmalion effects: existence, magnitude, and social importance. Harvard Educational Review, Vol. 16, $n^{\circ}$ 9, 37-41.

Sacerdote, B. (2001). Peer Effects with Random Assignment: Results for Dartmouth Roommates. Quarterly Journal of Economics, vol. 116, n. 2, 681-704.

Schindler, B. (2003). Educational Peer Effects. Quantile Regression Evidence from Denmark with PISA 2000 Data. Copenhage: Institute of Local Government Studies.

Slavin, R. (1987). Ability grouping and student achievement in elementary schools: A best-evidence synthesis. Review of Educational Research, n. 57, 293-336.

Slavin, R. (1990). Achievement effects of ability grouping in secondary schools: A best-evidence synthesis. Review of Educational Research, n. 60, 471-499.

Teddlie, Ch., S. Stringfield \& D. Reynolds (2000). "Context issues within school effectiveness research". En Ch. Teddlie y D. Reynolds (eds.), The international handbook of school effectiveness research. London: Routledge.

Thrupp, M., H. Lauder y T. Robinson (2002). School composition and peer effects. International Journal of Educational Research, n. 37, 483-504.

Treviño, E. et al. (2010). Factores asociados al logro cognitivo de los estudiantes de América Latina y el Caribe. Informe SERCE-LLECE, UNESCO. Santiago de Chile: UNESCO.

Valenzuela, J.P. (2008). Segregación en el Sistema Escolar Chileno: en la Búsqueda de una educación de calidad en un contexto de extrema desigualdad. En Transformaciones del Espacio Público, II Escuela Chile-Francia (pp. 131-156). Santiago de Chile: Facultad de Arquitectura y Urbanismo, Universidad de Chile.

Valenzuela, J.P., Bellei, C. \& De Los Ríos, D. (2009). Evolución de la segregación socioeconómica de los estudiantes chilenos y su relación con el financiamiento compartido. En Evidencias 
para Políticas Públicas en Educación (pp. 231-284). Santiago de Chile: FONIDE, Ministerio de Educación.

Valenzuela, J.P., C. Bellei \& D. De Los Ríos (2010). Segregación escolar en Chile. En S. Martinic \& G. Elacqua (eds.), Cambios en la gobernanza del sistema educativo chileno (pp.257-284). Santiago de Chile: UNESCO, Pontificia Universidad Católica de Chile.

Valenzuela, J.P., C. Bellei, A. Osses y A. Sevilla. (2009). ¿Qué explica las diferencias de resultados PISA Matemática entre Chile y algunos países de la OCDE y América Latina? En ¿Qué nos dice PISA sobre la educación de los jóvenes en Chile? (pp. 105-148). Santiago de Chile: Mineduc - OEI.

Wilkinson, I. (2002). Introduction: peer influences on learning: where are they? International Journal of Educational Research, n. 37, 395-401.

Wilkinson, I., J. Parr, I. Fung, J. Hattie \& M. Townsend (2002). Discussion: modeling and maximizing peer effects in school. International Journal of Educational Research, n. 37, 521-535.

Willms, J.D (2010). School Composition and Contextual Effects on Student Outcomes. Teachers College Record, vol. 112, n. 4, 1008-1037. 
